> Dans l'ensemble des pays européens, on observe une mortalité plus élevée parmi les personnes les moins favorisées socialement. Le niveau de ces inégalités varie toutefois fortement entre les pays et entre les hommes et les femmes. Les inégalités les plus faibles sont observées dans toutes les populations du Sud de l'Europe alors que les inégalités les plus importantes sont notées dans la plupart des pays de l'Est de l'Europe et les pays baltes. Ces variations semblent être attribuables en partie aux décès liés à la consommation de tabac ou d'alcool ou aux décès évitables, dus à l'absence d'intervention médicale appropriée. Ces résultats soulignent l'importance et l'urgence de conduire des politiques ambitieuses et efficaces de réduction de ces inégalités. <

Les inégalités sociales de santé constituent un problème majeur de santé publique. Pour de très nombreux problèmes de santé, elles touchent les personnes les moins favorisées socialement. II est important à la fois de quantifier de manière précise ces inégalités, mais aussi de déterminer dans quelle mesure elles peuvent être modifiées et réduites. Les études comparatives internationales peuvent aider à identifier les possibilités de réduction de ces inégalités. À ce titre, il est particulièrement intéressant de conduire des études en Europe étant donnée la variété de situations en terme d'histoire politique, culturelle, économique et épidémiologique dans les différents pays, mais aussi parce que les données de santé y sont généralement de bonne qualité.

Un projet précédent avait permis de comparer les inégalités sociales de mortalité et de morbidité dans les années 1980 en Europe de l'Ouest [1]. Nous présentons ici les résultats d'un projet plus récent et élargi à tous les pays d'Europe, jusqu'aux Pays Baltes. Disposer d'informations pour les pays d'Europe de l'Est permettra de déterminer si les pays ayant connu des périodes de troubles politiques, économiques ou des réformes importantes de leur système de santé se distinguent en termes d'inégalités sociales de santé. Nous nous intéresserons ici aux inégalités sociales de mortalité.

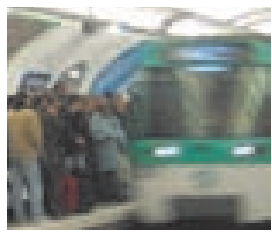

\section{Les données}

Les analyses ont porté sur plus de 3,5 millions de décès survenus au cours des années 1990 et au début des années 2000 dans une population de plus de 54 ithions de personnes vivant dans 16 pays européens et âgées de 30 à 74 ans. Les 16 pays considérés sont d'Ouest en Est et du Nord au Sud la Finlande, la Suède, la Norvège, le Danemark, l'Angleterre et le Pays de Galles (que l'on appellera par la suite simplement Angleterre), la Belgique, la Suisse, la France, I'Italie (Turin), l'Espagne (Madrid, Barcelone, Pays Basque), la Slovénie, la Pologne, la République Tchèque, la Hongrie, l'Estonie et la Lituanie. Les échantillons étaient généralement représentatifs de la population nationale, à l'exception de l'Italie (population de Turin), et de l'Espagne (population de Barcelone, de la région de Madrid et du Pays Basque) [2].

Le niveau d'études a été utilisé comme critère pour caractériser la situation sociale des individus, et quatre classes d'individus ont été définies: sans diplôme ou niveau d'études primaire (correspondant à environ 6 années d'études ou moins), niveau faible d'études secondaires (jusqu'à 9 années d'études environ), niveau élevé d'études secondaires (jusqu'à 11 années d'études environ) et niveau d'études supérieur (pour des diplômes supérieurs ou égaux au Baccalauréat). 


\section{Les inégalités sociales de mortalité diffèrent fortement entre les pays}

Les inégalités sociales ont été quantifiées à l'aide d'indices relatifs d'inégalités (IRI) [3]. Sans entrer dans les détails du calcul de cet indice, nous souhaitons en souligner une caractéristique importante. L'IRI est basé sur la position sociale relative des individus. Ainsi, ce qui est pris en compte n'est pas le fait d'avoir un diplôme supérieur mais de faire partie des $X \%$ les plus diplômés de la population. Comme l'on considère la position sociale relative, cela revient théoriquement à ordonner toute la population en fonction du niveau d'études. L'IRI s'interprète alors comme le risque de mortalité entre «la» personne la moins diplômée et «la» personne la plus diplômée de la population. Cette interprétation est bien entendu dans une certaine mesure théorique puisque ces deux personnes n'existent pas, l'information sur le niveau d'études étant disponible par catégorie. Toutes les personnes ayant le même niveau d'études se voient affecté la même situation sociale relative. Néanmoins, cet indice présente deux avantages majeurs : il est calculé à partir de l'ensemble de la population. Par ailleurs, comme il se fonde sur la position sociale relative, il permet de s'affranchir en partie de différences qui existeraient entre les pays dans le système éducatif, et de la signification des différents diplômes. L'interprétation de cet indice est la suivante: un indice relatif d'inégalités de 2 signifie que le risque de mortalité est multiplié par deux lorsque l'on va d'un bout à l'autre de l'échelle sociale. Par un raccourci de langage, on l'interprète aussi parfois en disant que le risque de décès est 2 fois plus élevé parmi les personnes les moins diplômées comparées aux personnes les plus diplômées.

Des inégalités sociales de mortalité sont observées dans l'ensemble des pays. L'IRI est toujours supérieur à $l$, ce qui signifie que le risque de décès est plus important parmi les personnes ayant un faible niveau d'études dans toute l'Europe aussi bien chez les hommes que chez les femmes (Figure 1). Toutefois, le niveau des inégalités sociales varie fortement d'un pays à l'autre. Les inégalités les plus faibles sont observées dans toutes les populations du Sud de l'Europe alors que les inégalités les plus importantes sont notées dans la plupart des pays de l'Est de l'Europe et les pays baltes. Ainsi chez les hommes, l'IRI est légèrement inférieur à 2 en Suède, ce qui signifie que la mortalité est moins de deux fois plus élevée parmi les hommes les moins diplômés que parmi les plus diplômés. Au contraire, cet indice est supérieur ou égal à 4 en Hongrie, République Tchèque ou Pologne, ce qui signifie que le risque de décès varie d'un facteur 4 ou plus d'une extrémité à l'autre de l'échelle sociale. Les inégalités sociales sont moins marquées chez les femmes mais l'on observe les mêmes variations géographiques que chez les hommes. Ainsi, l'IRI est inférieur à 1,5 dans les populations italienne et espagnole alors qu'il est proche de 3 en Hongrie, Pologne, République Tchèque et Lituanie.

\section{Le rôle de la consommation de tabac dans ces disparités}

Considérer la position sociale non pas comme un facteur de risque traditionnel mais comme le marqueur d'une exposition à des facteurs de risque de nature diverses (comportements de santé, utilisation du système de soins, facteurs environnementaux...) permet d'avancer dans la compréhension des inégalités sociales de santé $[4,5]$. II est malheureusement très difficile d'obtenir des données internationales de qualité documentant la distribution sociale de ces facteurs. Toutefois, documenter les inégalités sociales pour chacune des cause de décès est une première approche pour comprendre les disparités observées entre pays. Nous avons étudié plus particulièrement les décès liés à la consommation de tabac, ceux liés à la consommation d'alcool et les décès «évitables », c'est-à-dire ceux qui auraient pu être évités par une prise en charge et une intervention médicale appropriées. Le détail des causes de décès considérées pour ces trois groupes est indiqué dans les Figures 2 à 4 . II n'existe pas de définition unique pour la mortalité « évitable ». La définition retenue ici est basée sur des travaux antérieurs [6].

Le niveau des inégalités sociales de mortalité liée à la consommation de tabac varie fortement entre les pays européens (Figure 2). Chez les hommes, de fortes inégalités sont observées dans les pays d'Europe de l'Est mais aussi en Angleterre avec des IRI supérieurs à 5 . Chez les femmes, les inégalités sont comparables autour de 2-3

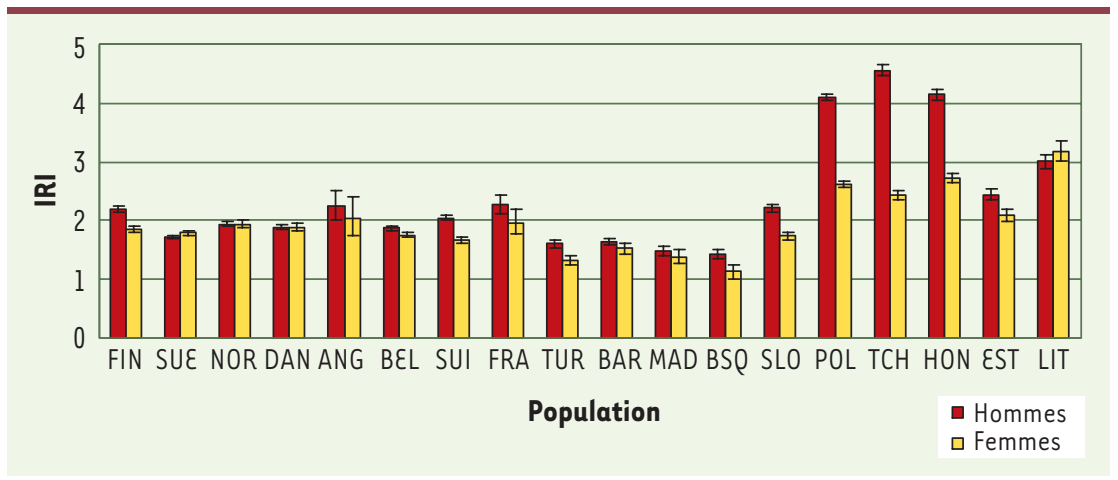

Figure 1. Indice relatif d'inégalités (IRI) et intervalle de confiance à $95 \%$ selon le niveau d'études pour la mortalité toutes causes par sexe et par population. FIN : Finlande, SUE: Suède, NOR: Norvège, DAN : Danemark, ANG : Angleterre et Pays de Galles, BEL: Belgique, SUI : Suisse, FRA: France, TUR: Turin, BAR: Barcelone, MAD : Madrid, BSQ : Pays Basque Espagnol, SLO: Slovénie, POL: Pologne, TCH : République Tchèque, HON : Hongrie, EST : Estonie, LIT : Lituanie. 


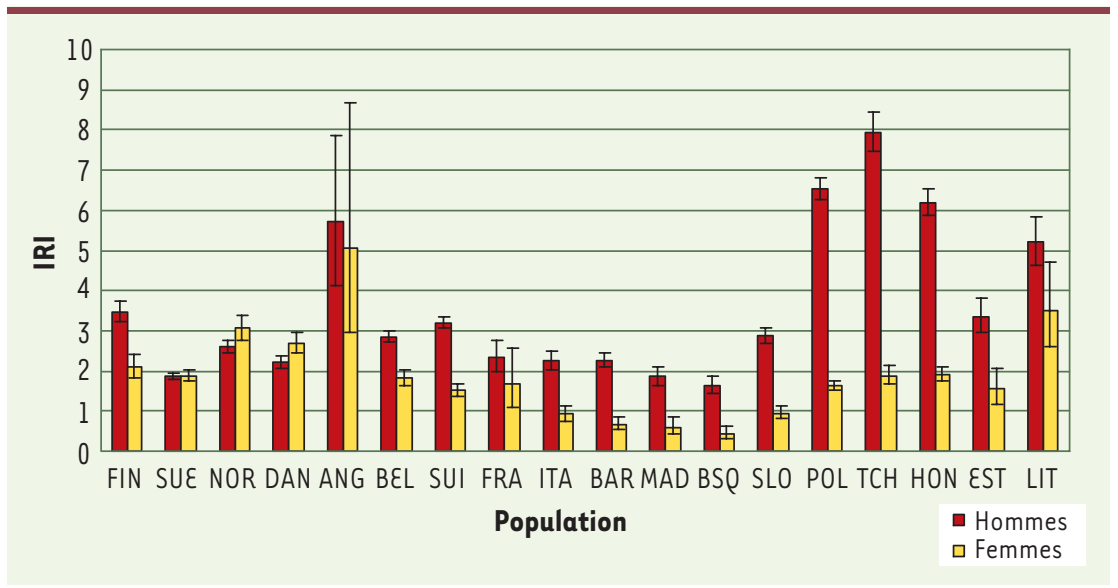

Figure 2. Indice relatif d'inégalités (IRI) et intervalle de confiance à $95 \%$ selon le niveau d'études pour la mortalité liée à la consommation de tabac par sexe et par population. Mortalité liée à la consommation de tabac: maladie pulmonaire obstructive chronique, cancer de la cavité buccale, du pharynx, de l'œsophage, du larynx, de la trachée, des bronches et du poumon.

dans la majorité des pays à l'exception de la Lituanie et de l'Angleterre où elles sont particulièrement élevées (et comparables à celles observées chez les hommes) et le Sud de l'Europe (populations italienne et espagnole, Slovénie) où les IRI sont inférieurs à 1 ce qui signifie que la mortalité y est plus élevée parmi les femmes les plus diplômées.

Il est intéressant d'analyser en parallèle les inégalités sociales de mortalité liées à la consommation de tabac et celles de la consommation de tabac, et de déterminer dans quelle mesure elles sont associées. Nous disposions de données sur la prévalence du tabagisme (être fumeur ou non) par sexe et niveau d'études pour la plupart des pays inclus dans cette étude (Figure 5). Ces données actualisent les connaissances sur ce sujet $[7,8]$. Elles montrent, dans les pays du Sud de l'Europe, de faibles différences sociales de consommation de tabac chez les hommes (I'IRI est proche de 1) alors que chez les femmes, les consommations les plus élevées sont toujours notées chez les femmes les plus diplômées (avec un IRI inférieur à l). Au contraire, dans les autres pays d'Europe de l'Ouest, le tabagisme est plus fréquent parmi les hommes et les femmes les moins diplômés. Ces inégalités sociales portant sur la consommation de tabac sont cohérentes avec les inégalités observées pour la mortalité liée à la consommation de tabac. Les inégalités sociales pour la mortalité liée à la consommation de tabac sont particulièrement élevées en Angleterre. C'est aussi dans ce pays que les inégalités de consommation de tabac sont parmi les plus élevées (Figure 5) ce que soulignaient déjà des données plus anciennes [8]. Certaines situations sont plus surprenantes. Ainsi, en Norvège, de fortes inégalités sociales de consommation de tabac sont

observées sans que les inégalités sociales pour la mortalité liée au tabac soient particulièrement élevées. Les informations sur la consommation de tabac ne concernaient que le statut tabagique, et cette information peu détaillée peut en partie expliquer les divergences observées entre les inégalités sociales de mortalité liée au tabac et les inégalités sociales de consommation de tabac.

La situation est plus difficile à interpréter dans les pays de l'Est de l'Europe ainsi que dans les pays baltes. En raison du manque de données historiques sur la consommation de tabac dans ces pays, il est difficile de comprendre la situation contradictoire qui prévaut dans certains pays comme la Lituanie ou la Hongrie avec de fortes inégalités sociales pour la mortalité liée au tabagisme mais de faibles inégalités sociales pour la consommation de tabac elle-même. De même, il est aussi difficile d'interpréter les fortes différences notées entre les hommes et les femmes dans le niveau des inégalités de mortalité liée au tabac.

\section{Autres facteurs explicatifs des disparités sociales de mortalité}

\section{Europe du Nord, Europe du Sud}

L'organisation du système de soins et de l'assurance médicale (avec la présence ou non d'une couverture

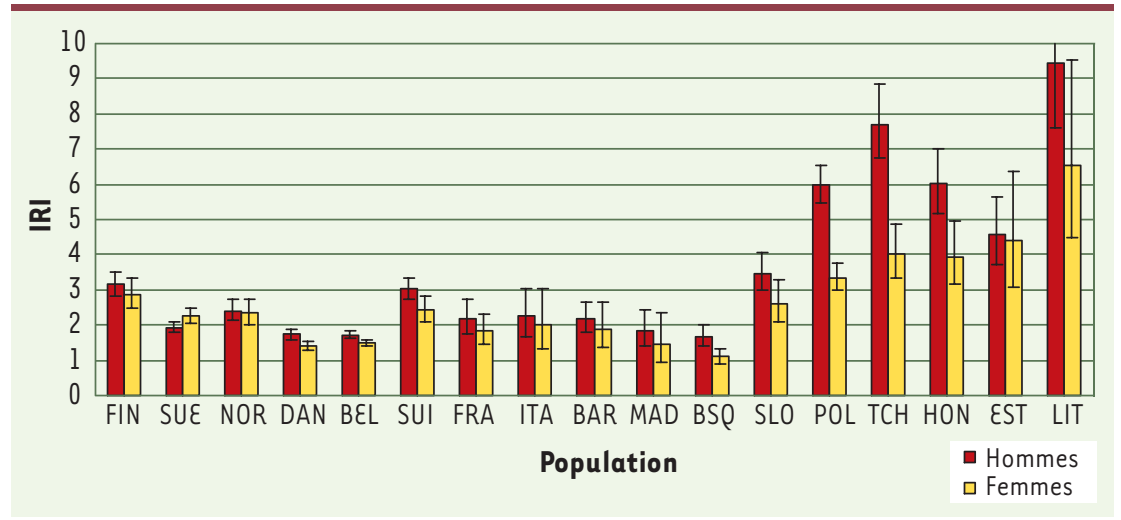

Figure 3. Indice relatif d'inégalités (IRI) et intervalle de confiance à $95 \%$ selon le niveau d'études pour la mortalité évitable par sexe et par population. Mortalité évitable : tuberculose et autres maladies infectieuses et parasitaires, cancer du col de l'utérus, cancer du sein, maladie de Hodgkin, leucémie, hypertension, maladie cérébrovasculaire, pneumonie ou grippe, appendicite, hernie, ulcère peptique, cholélithiase et cholécystite, complications de l'accouchement. Cette information n'était pas disponible pour l'Angleterre et le pays de Galles. 
sociale universelle) varie fortement entre les pays européens, et l'on peut se demander dans quelle mesure ces différences influent sur le niveau des inégalités sociales de mortalité. Les pays du Nord de l'Europe se caractérisent par un « Etat providence » avec un rôle important de l'état et des politiques de santé égalitaires avec une couverture santé universelle. En conséquence, les inégalités de revenus et le niveau de pauvreté y sont plus faibles que dans les autres pays. Au contraire, les pays du Sud de l'Europe comme l'Italie ou l'Espagne ont un système de couverture sociale moins généreux et moins universel. Les inégalités sociales de mortalité y sont pourtant plus réduites qu'ailleurs. Ainsi, un niveau minimal de couverture santé et de services publics, bien que nécessaire, ne suffit pas, à lui seul, à prévenir les inégalités sociales de mortalité. D’autres facteurs en lien avec le mode de vie sont aussi certainement à prendre en compte. La situation favorable observée en Europe du Sud s'explique probablement en partie par des facteurs culturels tels que le régime alimentaire méditerranéen ou la moins forte propension des femmes à fumer [7, 9]. II faut toutefois souligner que les populations d'Espagne et d'Italie considérées dans cette étude sont issues d'une zone géographique restreinte et souvent urbaine, et que les informations sur la mortalité ne sont pas représentatives de la situation dans l'ensemble du pays.

\section{Situation de l'Europe de l'Est et des pays Baltes}

Enfin, l'Europe de l'Est et les pays Baltes se détachent nettement du reste des pays tant les inégalités sociales de mortalité y sont importantes. C'est particuièrement vrai pour les décès évitables (chez les hommes seulement dans les pays de l'Est de l'Europe et chez les hommes et les femmes dans les pays Baltes) (Figure 3). Le rôle des déficiences dans le système de santé a déjà été évoqué pour expliquer les taux de mortalité élevés dans ces pays [3]. Nos résultats confirment donc que des inégalités sociales dans l'accès à un système de soins de qualité peuvent conduire à des inégalités sociales de mortalité. Par ailleurs, les inégalités sociales pour les décès liés à la consommation d'alcool sont extrêmement élevées dans ces pays, en particulier chez les hommes (Figure 4), ce qui souligne le rôle des consommations d'alcool à risque dans les inégalités sociales de mortalité. Ces pays se distinguent en effet par une consommation d'alcool extrêmement élevée, en particulier chez les hommes : quantité journalière élevée, binge drinking et consommation de produits contenant de l'alcool tels que l'eau de Cologne $[10,11]$, et par de substantielles inégalités sociales de consommation d'alcool. Plusieurs facteurs sont susceptibles d'expliquer ces différences de consommation : un faible support social, une absence de contrôle sur sa propre vie, des difficultés matérielles sans oublier une culture qui cautionne une consommation excessive d'alcool. De plus les différences sociales de consommation d'alcool sont plus marquées chez les hommes que chez les femmes, ce qui pourrait en partie expliquer les fortes différences notées entre hommes et femmes dans le niveau des inégalités sociales de mortalité liée à l'alcool [11].

La situation de la France se distingue par de fortes inégalités sociales pour la mortalité liée à la consommation d'alcool. Chez les femmes en particulier, les inégalités sont deux fois plus élevées que celles observées dans la majorité des pays de l'Ouest de l'Europe. Ces fortes inégalités sociales de consommation d'alcool et de mortalité liée à l'alcool, déjà identifées, se confirment sur des données plus récentes [12-15].

Plusieurs pays d'Europe de l'Est ainsi que les pays baltes ont récemment intégré l'Union Européenne. On peut se demander quelle sera l'influence de ce changement politique sur les inégalités sociales de mortalité dans ces pays. Les évolutions en termes d'espérance de vie sont mauvaises dans les pays baltes avec une faible augmentation de l'espérance de vie des personnes les plus favorisées socialement et une forte baisse chez les personnes les moins favorisées socialement [16]. Une consommation excessive d'alcool est certainement la cause de ces évolutions. Au contraire, l'espérance de vie augmente dans tous les groupes sociaux dans le reste de l'Europe, même si cette amélioration est plus marquée parmi les individus les plus favorisés socialement [16]. Ainsi, la situation observée dans les pays baltes avec une forte hausse des inégalités sociales d'espérance de vie et une détérioration de la santé parmi les personnes les plus défavorisées socialement peut laisser penser que la situation en termes d'inégalités sociales de mortalité s'améliorera beaucoup moins rapidement dans ces pays que dans les pays d'Europe de l'Est. Ces hypothèses nécessiteraient toutefois d'être vérifiées dans quelques années. Les résultats présentés ici quantifient les inégalités

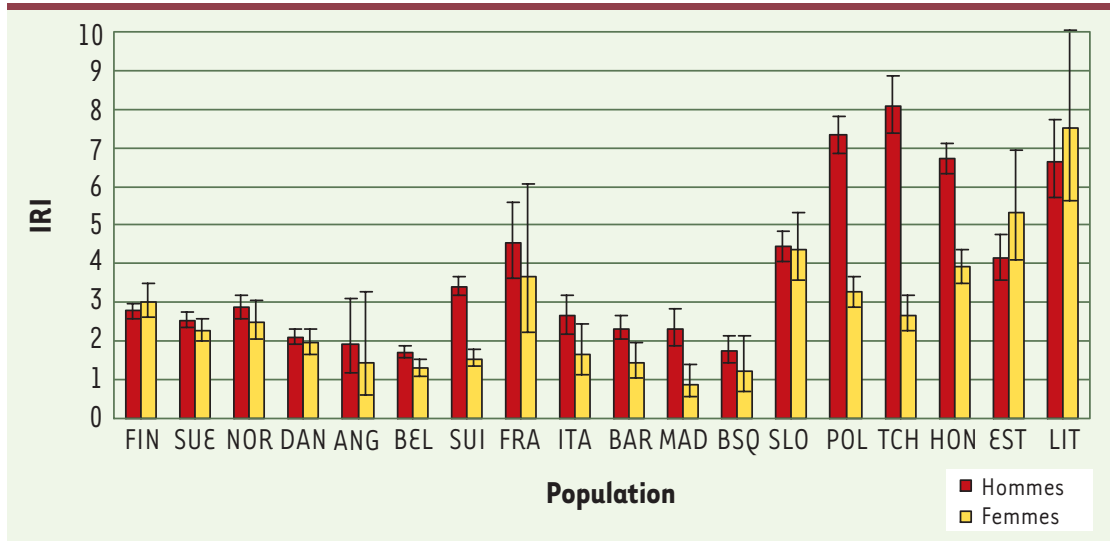

Figure 4. Indice relatif d'inégalités (IRI) et intervalle de confiance à $95 \%$ selon le niveau d'études pour la mortalité liée à la consommation d'alcool par sexe et par population. Mortalité liée à la consommation d'alcool: empoisonnement accidentel par l'alcool, psychose, dépendance, et abus alcoolique, cardiomyopathie, et cirrhose du foie et du pancréas. 


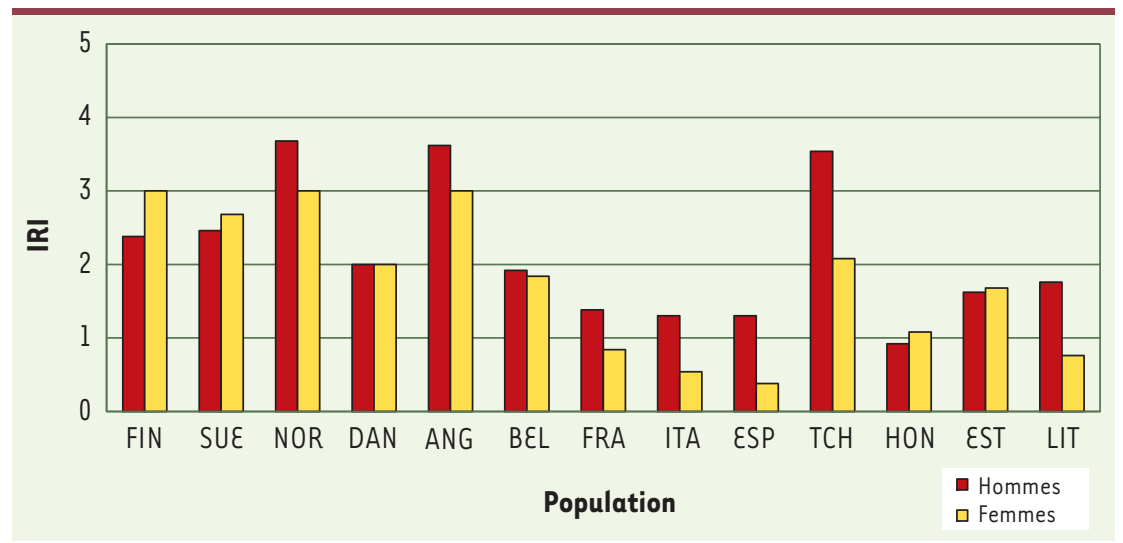

Figure 5. Indice relatif d'inégalités selon le niveau d'études associé à la consommation de tabac par sexe et par population. FIN : Finlande, SUE: Suède, NOR: Norvège, DAN : Danemark, ANG: Angleterre, BEL: Belgique, SUI : Suisse, FRA: France, ITA : Italie, ESP : Espagne, TCH : République Tchèque, HON : Hongrie, EST : Estonie, LIT : Lituanie.

sociales de manière relative c'est-à-dire sous la forme d'un rapport. II est aussi intéressant de quantifier les inégalités sociales de manière absolue, c'est-à-dire sous la forme d'un nombre de décès en excès. Les résultats de ces analyses (non présentées) vont dans le sens de ceux qui ont été présentés ici [2]. Ils soulignent aussi la situation très défavorable observée dans l'Est de l'Europe, où des taux de mortalité très élevés couplés à de fortes inégalités sociales entraînent un nombre excessif de décès parmi les personnes socialement défavorisées.

\section{Conclusion}

Des différences sociales de mortalité sont observées dans l'ensemble des pays européens, avec des taux de mortalité plus élevés parmi les personnes ayant un niveau d'études plus faible [17]. Toutefois, I'amplitude de ces différences varie fortement, même entre des pays relativement proches géographiquement. Ces résultats suggèrent un potentiel important de possibilité de réduction des inégalités sociales de mortalité et soulignent la nécessité et l'urgence de conduire des politiques ambitieuses et efficaces de réduction de ces inégalités. $\diamond$

\section{SUMMARY}

Socioeconomic inequalities in mortality in Europe In all European countries, the rates of death were higher in groups of lower socioeconomic status, but the magnitude of the inequalities between groups of higher and lower socioeconomic status was much larger in some countries than in others. Inequalities in mortality were small in some Southern European countries and very large in most countries in the eastern and Baltic regions. These variations among countries appeared to be attributable in part to causes of death related to smoking or alcohol use or amenable to medical intervention. These results imply that there is opportunity to reduce inequalities in mortality. Developing policies and interventions that effectively target the structural and immediate determinants of inequalities in health is an urgent priority for public health research. $\diamond$

\section{RÉFÉRENCES}

1. Kunst AE, Groenhof F, Mackenbach JP, Health EW. Occupational class and cause specific mortality in middle aged men in 11 European countries: comparison of population based studies. عU Working Group on Socioeconomic Inequalities in Health. Br Med J 1998 ; 316 : 1636-42.

2. Mackenbach JP, Stirbu I, Roskam AJ, et al. Socioeconomic inequalities in health in 22 European countries. N EnglJ Med 2008 ; 358 : 2468-81.

3. Velkova A, Wolleswinkel-van den Bosch JH, Mackenbach JP. The East-West life expectancy gap: differences in mortality from conditions amenable to medical intervention. Int J Epidemiol $1997 ; 26: 75-84$.

4. Bauman AE, Sallis JF, Dzewaltowski DA, Owen N. Toward a better understanding of the influences on physical activity: the role of determinants, correlates, causal variables, mediators, moderators, and confounders. Am J Prev Med 2002; 23 (suppl 2) : 5-14.

5. Singh-Manoux A, Clarke P, Marmot M. Multiple measures of socio-economic position and psychosocial health: proximal and distal measures. Int J Epidemiol $2002 ; 31$ : 1192-9.

6. Nolte $\varepsilon$, McKee M. Measuring the health of nations: analysis of mortality amenable to health care. Br Med J $2003 ; 327: 1129$

7. Huisman M, Kunst AE, Mackenbach JP. Educational inequalities in smoking among men and women aged 16 years and older in 11 European countries. Tob Control $2005 ; 14$ : 106-13.

8. Cavelaars $A \varepsilon$, Kunst $A \varepsilon$, Geurts JJ, et al. Educational differences in smoking: international comparison. BrMed J $2000 ; 320: 1102-7$.

9. Cavelaars AE, Kunst AE, Geurts JJ, et al. Differences in self reported morbidity by educational level: a comparison of 11 western European countries. J Epidemiol Community Health 1998 ; $52: 219-27$.

10. Kuntsche $\varepsilon$, Rehm J, Gmel G. Characteristics of binge drinkers in Europe. Soc Sci Med 2004 ; 59: 113-27.

11. Helasoja V, Lahelma $\varepsilon$, Prattala R, et al. The sociodemographic patterning of drinking and binge drinking in Estonia, Latvia, Lithuania and Finland, 1994-2002. BMC Publ Health 2007; $7: 241$.

12. Guignon N. Alcool et tabac. In : La société française. Données sociales. Paris : INSEદ, $1990: 254-7$

13. Cavelaars AE, Kunst AE, Mackenbach JP. Socioeconomic differences in risk factors for morbidity and mortality in the European Community: an international comparison. J Health Psychology $1997 ; 2: 353-72$

14. Desplanques G. L'inégalité sociale devant la mort. In : La société française. Données sociales. Paris : INSEE, $1993: 251-8$

15. Leclerc A, Lert F, Goldberg M. Les inégalités sociales devant la mort en Grande-Bretagne et en France. Soc Sci Med $1984 ; 19: 479-87$.

16. Shkolnikov VM, Andreev $\varepsilon M$, Jasilionis D, et al. The changing relation between education and life expectancy in central and eastern Europe in the 1990s. J Epidemiol Community Health $2006 ; 60: 875-81$.

17. Gilgenkrantz S. Les inégalités sociales face à la mortalité et aux incapacités. Med Sci (Paris) $2008 ; 24: 415-7$.
TIRÉS À PART

G. Menvielle 